\title{
Original
}

\section{Biomechanical Analysis of Poly Lactic-co-glycolic Acid Catheter Combined with Bone Marrow Mesenchymal Stem Cells and Extracellular Matrix Transplantation for Long Sciatic Nerve Defect Repair}

\author{
Chengdong Piao ${ }^{1)}$, Zhengwei Li $^{1)}$, Jie Ding ${ }^{2)}$ and Zhigang Qin ${ }^{3)}$ \\ 1) Department of Orthopedics, the Second Hospital of Jilin University, Changchun, China \\ 2) Department of Stomatology, the Affiliated Hospital of Changchun University of Traditional Chinese Medicine, Changchun, China \\ 3) Department of Neurosurgery, China-Japan Union Hospital of Jilin University, Changchun, China \\ (Accepted for publication, September 11, 2018)
}

\begin{abstract}
We aimed to study the biomechanical characteristics of sciatic nerve after transplantation with poly lactic-co-glycolic acid (PLGA) catheter combined with bone marrow mesenchymal stem cells (BMMSCs) and extracellular matrix (ECM) gel in rabbit model of sciatic nerve injury, so as to provide biomechanics and other basis for the clinical practice. The rabbit sciatic nerve injury model was used in this study. The rabbits with sciatic nerve injury received autologous nerve transplantation (ANT), PLGA catheter combined with BMMSCs transplantation and PLGA catheter combined with BMMSCs and ECM gel transplantation respectively and then correspondingly grouped into ANT group, PCBT group and PCBET group. Twenty-four weeks later, the sciatic nerves in each group were used for electrophysiological examination, histomorphological observation and tensile mechanical properties test. Amplitude (AMP) and motor nerve conduction velocity (MNCV) values of the sciatic nerves in PCBET group were remarkably higher than those in the PCBT group and ANT group with statistical significance $(P<0.05)$. The tensile elastic limit stress, elastic limit strain, maximum stress and strain in PCBET group were all larger than those in the PCBT group and ANT group with a significant difference $(P<0.05)$. PLGA catheter combined with BMMSCs or PLGA catheter combined with BMSCs and ECM gel can restore the elasticity and toughness of the injured sciatic nerve at some extent, and has obvious recovery effect on the function of the injured sciatic nerves.
\end{abstract}

Key words: Sciatic nerve defect, Autologous nerve graft, Jointgraft, Mechanical properties

\section{Introduction}

Tissue engineering brings new hope for obtaining tissue and graft through surgical operations ${ }^{1)}$. Polylactic acid (PLA), polyglycolic acid (PGA) and their copolymers (PLGA) are a class of materials most widely researched and applied in tissue engineering at present ${ }^{2}$. The scholars have made a large number of researches on the peripheral nerve injury by PLGA catheter transplantation and so on ${ }^{3-5)}$. Huang et $\mathrm{al}^{6)}$ used lamin-modified PLGA film to repair the injured peripheral nerve and found that as a core of the extracellular matrix protein, lamin can significantly improve the adhesion and affinity of the Schwann cells, which is conducive to the peripheral nerve regeneration. Moore et $\mathrm{al}^{7}$ processed PLGA into multipass nerve scaffold and implanted the multipass nerve scaffold combined with the Schwann cells into the rat transverse spinal cord. 30 days later, the spinal cord axons regenerated.

Bone marrow mesenchymal stem cells (BMSCs), a cluster of adult pluripotent stem cells, are important cell sources for cell therapy and tissue engineering repair at present ${ }^{8)}$. The scholars have made a lot of efforts on the treatment of nerve injury using BMSCs transplantation. Kurwal et $\mathrm{al}^{9)}$ implanted the BMMSCs isolated from the rat bone marrow into fibrin catheter and used them to repair the $12 \mathrm{~mm}$ defect in rat

Correspondence to: Dr. Zhigang Qin, Department of Neurosurgery, ChinaJapan Friendship Hospital, Jilin University, No.126 Xiantai Avenue, Changchun 130031, Jilin Province, China; Tel: +86 431 88706136; Fax: +86 431 88706136; E-mail: doczhigangqin@126.com sciatic nerve. The results showed that the curative effect of the fibrin catheter combined with BMMSCs is significantly better than that of simple fibrin catheter group. Zhou et $\mathrm{al}^{10)}$ treated $10 \mathrm{~mm}$ defect in rat sciatic nerve using the acellular nerve scaffold combined with BMMSCs and stem cells (SCs), and found that the combination of BMMSCs and SCs had better effect 16 weeks later than single BMMSCs or SCs transplantation. However, the previous studies have never involved the biomechanical properties analysis on the sciatic nerve repaired by PLGA catheter combined with BMMSCs and extracellular matrix gel (ECM) transplantation ${ }^{9,10)}$. The destruction of soft tissues and organs in animals will change their biomechanical proper$\operatorname{ties}^{11,12)}$.

Therefore, we hypothesized that the biomechanical properties of the sciatic nerve group will change after its structure of sciatic nerve is destructed, and the joint transplantation of PLGA catheter, BMSCs, and ECM will have certain effects on the repair of the damaged tissue structure, and the biomechanical properties of the sciatic nerve will get certain recovery. In view of this, the authors tested the electrophysiology, tensile mechanics, and histomorphology of the sciatic nerves in the animal model with sciatic nerve defect after joint graft of PLGA catheters and BMSCs or joint graft of PLGA catheters, BMSCs, and ECM,

Using biomechanical index to judge the therapeutic effects of PLGA catheter combined with BMMSCs transplantation and PLGA catheter combined with BMMSCs and ECM transplantation on the repair of sci- 

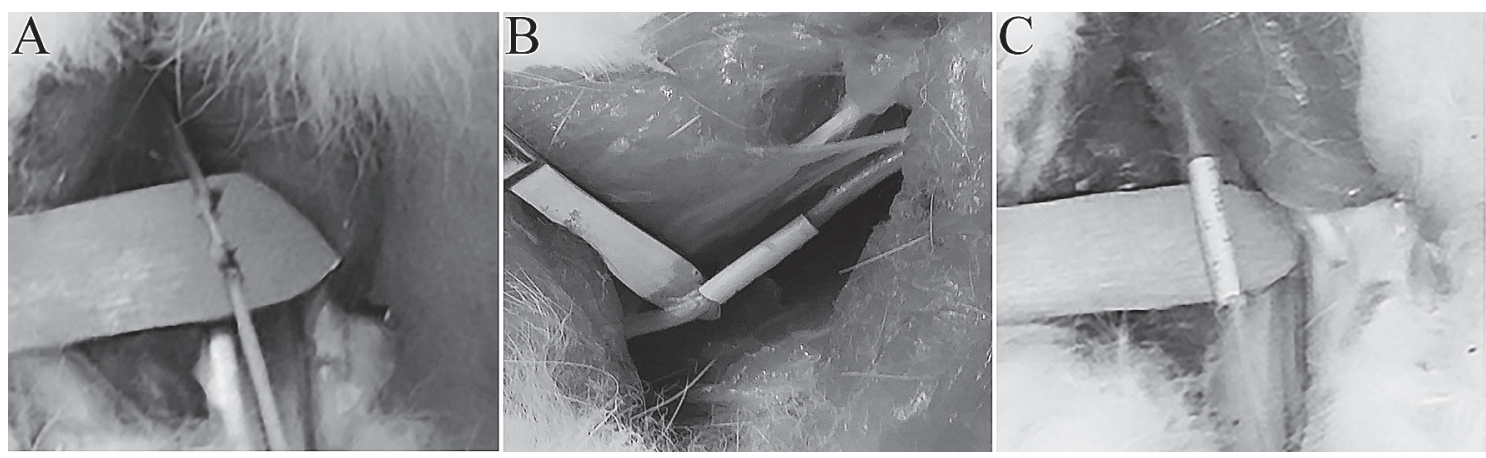

Figure 1. Different groups with different surgeries. A: Rabbits in the ANT group with autologous nerve transplantation. B: Rabbits in the PCBT group were treated with PLGA catheter combined with BMMSCs transplantation. C: Rabbits in the PCBET group were treated with PLGA catheter combined with BMMSCs and ECM gel transplantation.

atic nerve injury could provide biomechanical basis for the clinical repair of sciatic nerve injury.

\begin{abstract}
Materials and Methods
Animals

Sixty healthy female Japanese white rabbits, aged 6 month and weighing 2.2-2.4 kg, were provided by Changchun High-tech Animal Experimental Center (license number: SCXK (Ji) 2003-0004). Rabbits were fed in cages with full grains feed. All rabbits were housed in a circumstance of constant temperature $22-24{ }^{\circ} \mathrm{C}$, relative humidity $54-66 \%$, natural lighting and well ventilated and free to drinking and food. This study was carried out in strict accordance with the recommendations in the Guide for the Care and Use of Laboratory Animals of the National Institutes of Health. The animal use protocol has been reviewed and approved by the Institutional Animal Care and Use Committee (IACUC) of Jinlin University.
\end{abstract}

\section{Preparation of PLGA catheter}

PLGA (Changchun Sinobiomaterials Co. Ltd., Changchun, Jilin, China) was dissolved in dichloromethane at a volume ratio of 70:30. $\mathrm{NaCl}$ with particle size of 200-300 micron was added as porogen (the mass ratio of PLGA and $\mathrm{NaCl}$ was 1:9) and mixed. The mixture was poured into a preset mould with inner diameter $1.6 \mathrm{~mm}$, outer diameter $1.8 \mathrm{~mm}$ and length $40 \mathrm{~mm}$. The mixture in the mould was naturally volatilized at room temperature in a fume cupboard. 96 hours later, the mold was released to pick out the PLGA and $\mathrm{NaCl}$ cylindrical catheter. After dried in a vacuum drying oven at $37{ }^{\circ} \mathrm{C}$ for $48 \mathrm{~h}$, the PLGA and $\mathrm{NaCl}$ cylindrical catheter was soaked into $800 \mathrm{ml}$ deionized water for 96 hours. During this period, the deionized water was changed every 4 hours. Then the PLGA and $\mathrm{NaCl}$ cylindrical catheter was dried in a dry box at $37{ }^{\circ} \mathrm{C}$ for 48 hours, followed by disinfection with $36{ }^{\circ} \mathrm{C}$ ethylene oxide for 12 hours. Then PLAG catheters were prepared for use. Before the experiment, the PLGA and $\mathrm{NaCl}$ catheters were cut into length 30 $\mathrm{mm}$ for use by the S-5 aseptic plastic scalpel which was produced by Xuyi Union Huai'an Yikang Medical Products Co., Ltd. (Xuyi, Jiangsu, China). Seventy pieces of $\mathrm{NaCl}$ and PLGA catheters were prepared for use.

\section{Preparation of sciatic nerve defect animal model and grouping}

The experimental rabbit was fixed on the animal operating table and anesthetized with $6 \%$ chloral hydrate $(6 \mathrm{ml} / \mathrm{kg})$ by intraperitoneal injection. After successful anesthesia, a midline incision was made on the posterior part of the rabbit left thigh. The left sciatic nerve was exposed and isolated layer by layer. At the $3 \mathrm{~mm}$ away from the inferior margin of the piriform muscle, a $30 \mathrm{~mm}$ sciatic nerve was excised to prepare the model of $30 \mathrm{~mm}$ sciatic nerve defect. Then, the prepared animals were randomly divided into 3 groups: rabbits with sciatic nerve defect treated with autologous nerve transplantation (ANT group, $N=20$ ), rabbits with sciatic nerve defect treated with PLGA catheter combined with BMMSCs transplantation (PCBT group, $N=20$ ), and rabbits with sciatic nerve defect treated with PLGA catheter combined with BMMSCs and ECM transplantation (PCBET group, $\mathrm{N}=20$ ).

\section{Treatments}

Rabbits in the ANT group were treated with autologous nerve transplantation. Under XT-X-6A surgical microscope (Zhenjiang Xintian Medical Instrument Co. Ltd., China), the autologous sciatic nerve cut off from the rabbit was implanted back into the sciatic nerve by suturing the epineurium of the ends using 9-0 noninvasive suture line (Qingdao Nike Medical Material Co. Ltd., China). Each end was sutured by four punctures (Fig. 1A), and then the muscles and skin were closed.

Rabbits in the PCBT group were treated with PLGA catheter combined with BMMSCs transplantation. BMSCs were produced by Shanghai Yanyi Biotech Co. Ltd (Shanghai, China). The BMMSCs were cultured and subcultured in strict accordance with the instructions provided by the manufacturer. When the cells grew to $90 \%$ confluence, they were passaged at a density of $2 \times 10^{4} / \mathrm{cm}^{2}$ at a circumstance of $95 \%$ air and $5 \%$ $\mathrm{CO}_{2}$ at $37{ }^{\circ} \mathrm{C}$ after digestion by $0.05 \%$ trypsin. Cells were passaged every 5 days at a ratio of 1:2. The medium was changed every 3 days. The shape of rabbit BMMSCs was fibroblast-like in the early stage. We also found that there were several cell components in bone marrow. In addition to the differentiated cells such as stroma cells, there were also two kinds of pluripotent stem cells, hematopoietic stem cells and mesenchymal stem cells. Those with the phenotype of bone marrow mesenchymal stem cells were used in this study. Under the surgical microscope, the broken nerve ends were inserted into the both ends of the 30 mm PLGA nerve catheter, respectively. Then the epineurium and the PLGA nerve catheter wall were fixed with 9-0 noninvasive suture line (Qingdao Nike Medical Material Co., Ltd.) and sutured by four punctures at both ends. Then $1 \times 10^{9}$ cells/ 1 BMMSCs at the fifth generation grown in a $5 \% \mathrm{CO}_{2}$ saturated humidity incubator at $37{ }^{\circ} \mathrm{C}$ were injected into the PLGA nerve catheter using a micro injector (Fig. 1B). After that, the muscles and skin were closed.

Rabbits in the PCBET group were treated with PLGA catheter combined with BMMSCs and ECM gel transplantation. The extracellular Matrix Gel was produced by BD (California, USA). Under the surgical microscope, the broken ends of the sciatic nerve were inserted into the both ends of the $30 \mathrm{~mm}$ PLGA nerve catheter, respectively. Then the 
Chengdong Piao et al:: Rabbit Sciatic Nerve Injury, Joint Graft of PLGA, BMSCs, and ECM, Biomechanics

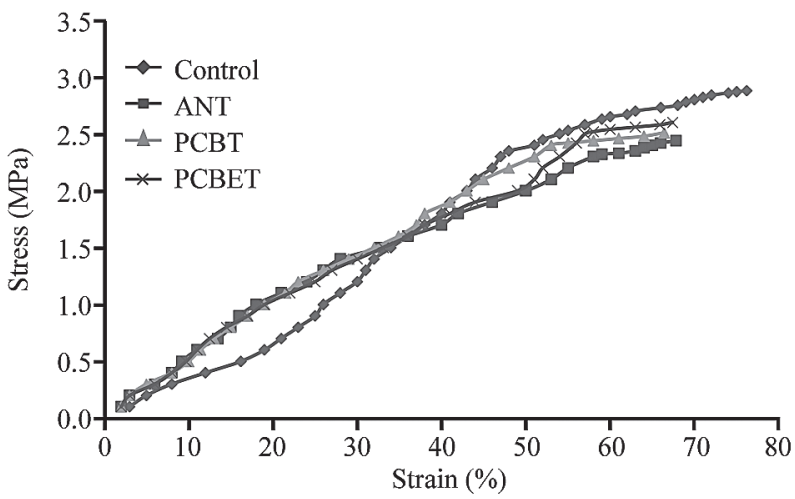

Figure 2. The stress-strain curves of the sciatic nerve in all the four groups. The ordinate represents the stress and the abscissa represents the strain. The establishment of the formula of stress-strain functional relation. From the stress-strain curves, the formula of stress-strain functional relation were established as follows:

The control group: $\sigma(\varepsilon)=2.9299 \mathrm{e}^{5}-10.5160 \mathrm{e}^{4}+4.6152 \mathrm{e}^{3}-30.2386 \mathrm{e}^{2}-0.2936+0.7472$;

The ANT group: $\sigma(\varepsilon)=4.9598 \mathrm{e}^{5}-21.7445 \mathrm{e}^{4}+35.7006 \mathrm{e}^{3}-0.9259^{2}+25.134$

The PCBT group: $\sigma(\varepsilon)=-0.7992 \mathrm{e}^{5}+4.6109 \mathrm{e}^{4}-2.6227 \mathrm{e}^{3}+0.4126 \mathrm{e}^{2}+1.1828$

The PCBET group: $\sigma(\varepsilon)=0.9285 \mathrm{e}^{5}-6.5147 \mathrm{e}^{4}+17.3074 \mathrm{e}^{3}+602544 \mathrm{e}^{2}+1.9244$

epineurium and the PLGA nerve conduit wall were fixed and sutured with 9-0 noninvasive suture line (Qingdao Nike Medical Material Co., Ltd.) by four punctures at both ends. The fifth generation of rabbit BMMSCs $\left(1 \times 10^{9}\right.$ cells $\left./ 1\right)$ grown in a $5 \% \mathrm{CO}_{2}$ saturated humidity incubator at $37^{\circ} \mathrm{C}$ and extracellular matrix gel $\left(1 \times 10^{9} / 1\right)$ were injected into the PLGA nerve catheter using a micro injector (Fig. 1C). Then the muscles and skin were closed.

After surgery, the incision on each rabbit was rinsed with gentamicin to avoid infection. The rabbit limb was not externally fixed. When the rabbits were awoken from anesthesia, they were separately fed in cages, and each rabbit received intraperitoneal injection of penicillin $10,000 \mu /$ $\mathrm{kg}$ twice daily for 7 days. The skin incision was disinfected with $75 \%$ alcohol once daily for 7 days. The rabbit cage padding was kept dry all the time.

\section{Electrophysiological test of sciatic nerve}

At 24 weeks after operation, electrophysiological test was carried out on the sciatic nerve of the experimental side (left) of each rabbit using NIM-Neuro Type 2 electromyograph (Medtronic Company, Minneapolis, Minnesota, USA). Rabbits were anesthetized with $10 \%$ chloral hydrate $(10 \mathrm{ml} / \mathrm{kg})$ by intraperitoneal injection. After the surgical field was disinfected, the rabbit was on prone position to expose the left and right sciatic nerve stem. The co-core needle electrode was punctured into the soleus muscle (M) as a recording electrode, while the grounding wire was fixed on the edge of the wounded skin with an alligator clips. A super stimulation of $50 \mathrm{~mA}$ current was applied on the parallel stimulating electrodes which were put on the proximal anastomotic stoma of the ischial tuberosity level (P) and the distal anastomotic stoma of the sciatic nerve branches (D) respectively to induce two action potentials. The electromyograph automatically displayed the latency and amplitude of action potential (AMP). The distance between the two electrodes was measured with a vernier caliper and input the instrument. The electromyograph could also automatically display the value of motor nerve conduction velocity (MNCV). Twenty rabbits in the experimental group were randomly selected to detect the right sciatic nerves by the same method as the normal control group.

After the electrophysiological test, using the anastomotic stoma of the rabbit sciatic nerve as the midpoint, a length of about $20 \mathrm{~mm}$ sciatic
Table 1 The electrophysiological results of the rabbit sciatic nerve in each group $(n=20)$.

\begin{tabular}{ccc}
\hline Groups & AMP $(\mathrm{Mv})$ & MNCV $(\mathrm{m} / \mathrm{s})$ \\
\hline Control & $13.4 \pm 1.0^{\mathrm{a}}$ & $54.3 \pm 1.2^{\mathrm{a}}$ \\
ANT & $10.5 \pm 0.8^{\mathrm{b}}$ & $51.0 \pm 1.08^{\mathrm{b}}$ \\
PCBT & $11.5 \pm 0.7^{\mathrm{c}}$ & $53.0 \pm 01.0^{\mathrm{c}}$ \\
PCBET & $12.5 \pm 0.7^{\mathrm{d}}$ & $52.2 \pm 1.6^{\mathrm{d}}$ \\
\hline
\end{tabular}

Note: Data are shown as mean \pm SD. ${ }^{a} P<0.05$, vs. PCBT group and PCBET group; ${ }^{\mathrm{b}} P<0.05$, vs. PCBT group and $\mathrm{PCBET}$ group; ${ }^{\mathrm{c}} P<0.05$, vs. PCBT group and PCBET group; ${ }^{\mathrm{d}} \mathrm{P}<0.05$, vs. control group.

nerve from each experimental side (left) was cut off as test samples $(N=20)$. Twenty right sciatic nerves were randomly selected from each group to serve as the normal control group. All nerve specimens were kept in physiological saline for use.

\section{Tensile test}

MODEL55100 type automatically controlled electronic universal test machine produced by Changchun Test Machine Institute Group (Changchun, Jilin, China) was used in this study for the tensile test. The universal test machine equipped with a temperature adjustable thermostat which could be adjusted from $-30{ }^{\circ} \mathrm{C}$ to $250{ }^{\circ} \mathrm{C}$. CGA- 5 type microscope produced by Changchun the Third Optical Instrument Factory (Changchun, Jilin, China) was employed to measure the length and diameter of sciatic nerve. The initial length and diameter of the sciatic nerve specimens in the control group, the ANT group, the PCBT group and the PCBET group were all $20 \mathrm{~mm}$ and 1.52-1.55 mm, respectively. According to the method described in the literature ${ }^{13,14}$, each sciatic nerve specimen was pretreated for the following experiments. The experimental temperature was controlled within $36.5 \pm 1.0^{\circ} \mathrm{C}$. Each sciatic nerve specimen was clamped in the soft tissue holder, and a tensile load was applied to the specimen at a speed of $5 \mathrm{~mm} / \mathrm{min}$. In order to maintain the humidity of the specimen during the test, the normal saline was sprayed continuously to the sciatic nerve specimen. After the test, the computer automatically output the elastic limit strain, the maximum strain, the elastic limit stress, the maximum stress, the elastic limit load, the maximum load and stress-strain curves.

\section{Observation of tissue morphology}

One sciatic nerve specimen from the same part was randomly selected from each group to make frozen sections. Prepared sections were treated by routine manners and stained with hematoxylin and eosin (H\&E). After staining, sections were dehydrated through increasing concentrations of ethanol and xylene. The species were observed by light microscope (BX51, Olympus, Tokyo, Japan) to find the change of the transect of nerve, including axons, nerve cells, myelin sheath, and nerve base-membrane.

\section{Statistical analysis}

The measurement data were expressed as mean \pm standard deviation (SD) and analyzed using SPSS16.0 software package (SPSS, Chicago, IL, USA). The difference of data between groups was analyzed using one-way ANOVA and Scheffe method. $P<0.05$ was regarded as significant difference. The formula of stress-strain functional relation of each group was established by mathematical regression analysis. 
J.Hard Tissue Biology Vol. 27(4): 327-332, 2018

Table 2 The tensile test results of the rabbit sciatic nerve in each group $(n=20)$.

\begin{tabular}{lllllll}
\hline Groups & Maximum load (N) & $\begin{array}{l}\text { Maximum stress } \\
(\mathrm{MPa})\end{array}$ & Maximum strain (\%) & $\begin{array}{l}\text { Elastic limit strain } \\
(\%)\end{array}$ & $\begin{array}{l}\text { Elastic limit load (N) } \\
(\mathrm{MPa})\end{array}$ & $\begin{array}{l}\text { Elastic limit stress } \\
(\mathrm{M})\end{array}$ \\
\hline Control & $5.99 \pm 0.21^{\mathrm{d}}$ & $2.88 \pm 0.12^{\mathrm{d}}$ & $76.2 \pm 1.26^{\mathrm{d}}$ & $40.6 \pm 1.16^{\mathrm{d}}$ & $2.74 \pm 0.13^{\mathrm{d}}$ & $1.31 \pm 0.06^{\mathrm{d}}$ \\
ANT & $5.13 \pm 0.06^{\mathrm{a}}$ & $2.44 \pm 0.03^{\mathrm{a}}$ & $67.8 \pm 0.66^{\mathrm{a}}$ & $32.7 \pm 0.71^{\mathrm{a}}$ & $2.38 \pm 0.12^{\mathrm{a}}$ & $1.18 \pm 0.06^{\mathrm{a}}$ \\
PCBT & $5.25 \pm 0.07^{\mathrm{b}}$ & $2.51 \pm 0.03^{\mathrm{b}}$ & $66.4 \pm 0.83^{\mathrm{b}}$ & $33.2 \pm 0.92^{\mathrm{b}}$ & $2.52 \pm 0.09^{\mathrm{b}}$ & $1.24 \pm 0.04^{\mathrm{b}}$ \\
PCBET & $5.32 \pm 0.11^{\mathrm{c}}$ & $2.59 \pm 0.04^{\mathrm{c}}$ & $67.4 \pm 0.69^{\mathrm{c}}$ & $34.9 \pm 1.24^{\mathrm{c}}$ & $2.62 \pm 0.09^{\mathrm{c}}$ & $1.29 \pm 0.04^{\mathrm{c}}$ \\
\hline
\end{tabular}

Note: Data are shown as mean \pm SD. ${ }^{\mathrm{a}} P<0.05, v s$. PCBT group and PCBET group; ${ }^{\mathrm{b}} P<0.05, v s$. PCBT group and PCBET group; ${ }^{\mathrm{c}} P<0.05, v s$. PCBT group and PCBET group; ${ }^{\mathrm{d}} P<0.05, v s$. control group.
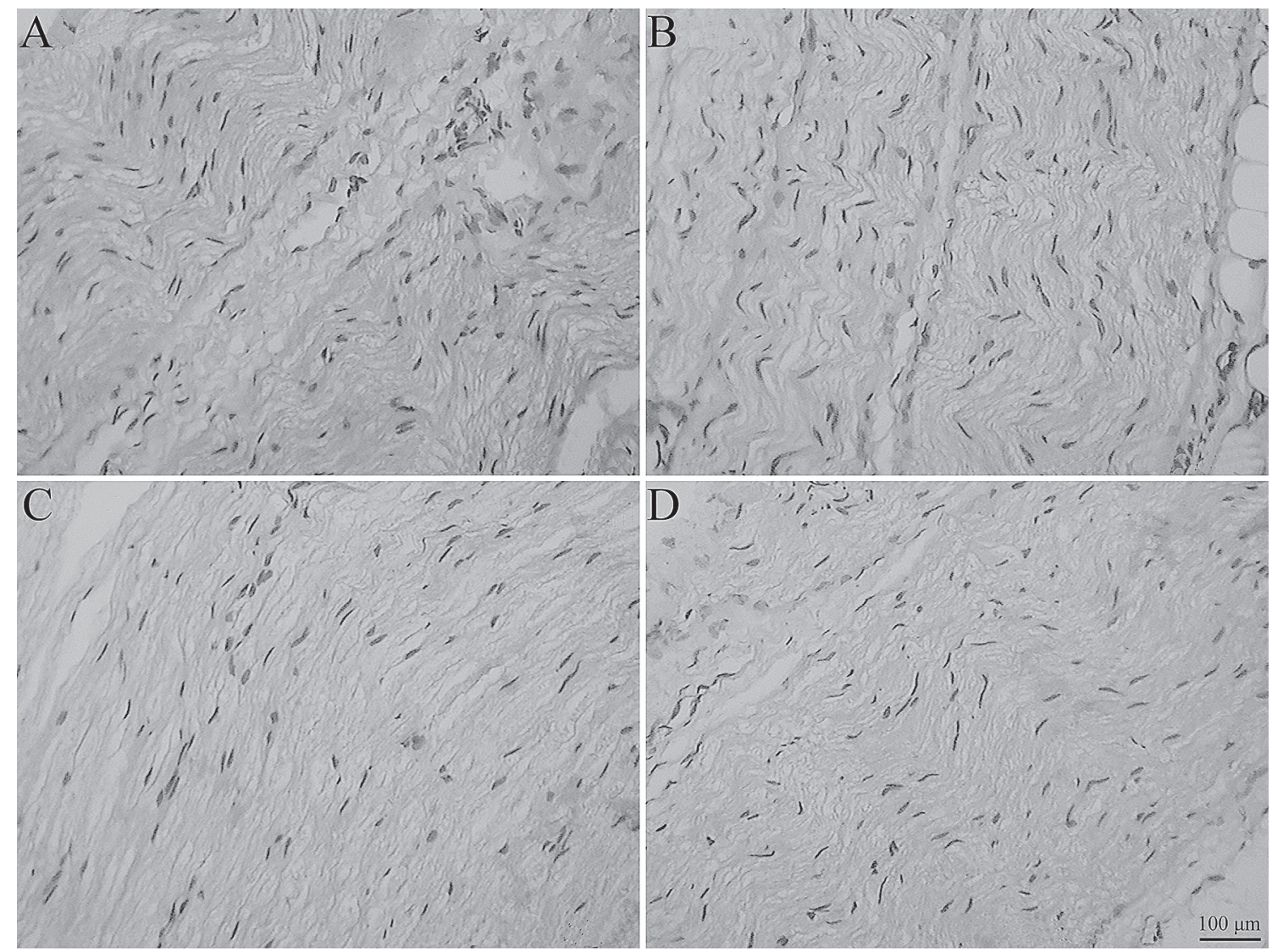

Figure 3. The cross-section of rabbit sciatic nerves observed under a light microscrope (HE staining). Scale bar $=100$. A: A representative of the rabbit sciatic nerves in the control group; B: A representative of the rabbit sciatic nerves in the ANT group; C: A representative of the rabbit sciatic nerves in the PCBET group; D: A representative of the rabbit sciatic nerves in the PCBT group.

\begin{abstract}
Results

\section{Electrophysiological results}

The electrophysiological results of the sciatic nerve in each group were shown in Table 1. The electrophysiological test showed that the AMP and MNCV values of the sciatic nerve in the ANT, PCBT and PCBET groups were significantly lower than those in the control group $(P<0.05)$. Howover, the AMP and MNCV values of the sciatic nerve in the PCBET group were both markedly higher than those in the ANT and PCBT groups with significant difference $(P<0.05)$. In addition, the AMP and MNCV values of the sciatic nerve in the PCBT group were also notably higher than those in the ANT group $(P<0.05)$.
\end{abstract}

\section{Tensile results of the sciatic nerve specimens}

The tensile results of the rabbit sciatic nerve specimens in each group were shown in Table 2. The maximum load, maximum stress, elastic limit strain, elastic limit load and elastic limit stress of group
PCBT and PCBET group were greater than those of group ANT $(P<0.05)$, but the maximum strain of ANT group was not significantly different from that of group PCBT and PCBET group $(P>0.05)$.

\section{The stress-strain curve of the sciatic nerve specimens}

The stress-strain curves of the sciatic nerves in all the four groups were shown in Fig. 2. According to Fig. 2, the stress-strain curve of the control group was almost exponential when the strain of the sciatic nerve rose from 0 to $16.2 \%$ and approximately linear when the strain rose from $16.3 \%$ to $40.6 \%$. However, when the strain was increased from $40.7 \%$ to $76.2 \%$, the deformation of the sciatic nerve in the control group increased greatly and the nearly no loading capacity of the specimen left which tended to be damaged. In the ANT group, the stressstrain curve was basically exponential when the sciatic nerve strain was increased from 0 to $9.2 \%$ and approximately linear when the strain was increased from $9.3 \%$ to $32.7 \%$. But when the strain rose from $32.8 \%$ to 
$67.8 \%$, the specimen had large deformation with extremely low bearing capacity and tended to be destroyed. When the strain of the sciatic nerve in the PCBT group was increased from 0 to $9.7 \%$, the stress-strain curve basically had an exponential relationship, but it was similar to the linear functional relation when the strain increased from $9.8 \%$ to $33.1 \%$. When the strain was increased from $33.4 \%$ to $66.4 \%$, the specimens in the PCBT group had great deformation with very low bearing capacity which led to the specimens to be destroyed. The stress-strain curve of the PCBET group was almost exponential as the sciatic nerve strain was increased from 0 to $11.4 \%$ and approximately linear as the strain was increased from $11.5 \%$ to $34.9 \%$. In the PCBET group, the sciatic nerve specimens had large deformation and extremely low bearing capacity when the strain was elevated from $35 \%$ to $67.4 \%$, which led to the specimens almost destroyed.

\section{Morphological observation of the sciatic nerve specimens}

The sciatic nerve specimens of each group were also used for the histopathological examination. The histopathological examination revealed that the rabbit sciatic nerve fibers in normal control group arranged orderly and the contents of nerve fiber such as axon had clear form; in addition, the axons were surrounded by myelin sheath (Fig. $3 \mathrm{~A})$. In the ANT group, fiber axons were observed beside the Schwann cells in the rabbit sciatic nerve, and a large number of early myelination was visible at the distal end (Fig. 3B). The sciatic nerve had a good myelinization in the PCBET group; good nerve regeneration and orderly arrangements of the nerve fibers were also observed (Fig. 3C). The sciatic nerve regeneration was fairly complete in the PCBT group, with a good myelinization; the nerve fibers were arranged orderly (Fig. 3D).

\section{Discussion}

Electrophysiological index is one of the important methods to judge the recovery effect of peripheral nerve injury in human or animals. In this study, the electrophysiological test on the rabbit sciatic nerves in each group showed that the MNCV and AMP values in the rabbits with sciatic nerve injury receiving PLGA catheter combined with BMMSCs and ECM transplantation (PCBET group) were notably higher than those in the rabbits receiving autograft nerve transplantation (ANT group) or receiving PLGA catheter combined with BMMSCs transplantation (PCBT group) with significant difference $(P<0.05)$, suggesting that the injured sciatic nerve treated with PLGA catheter combined with BMMSCs and ECM transplantation got functional recovery to some extent.

Optical microscope observation on the microstructure of the sciatic nerves after transplantation repair is an important way to compare the recovery effects of various transplanting anastomoses on repairing the sciatic nerve injury. In this study, we found the sciatic nerve regeneration in the PCBET group was complete and the myelinization was well, the nerve fibers arranged orderly prompting that PLGA catheter combined with BMMSCs and ECM transplantation is conducive to the recovery of the injured animal nerve fibers such as sciatic nerve fiber. Although Bodian and Kluver-barrera double staining was not conducted in this study, HE stain of the sciatic nerve was enough to show that receiving PLGA catheter combined with BMMSCs and ECM transplantation were effective to repair the injury sciatic nerve.

From the stress-strain curves of the sciatic nerves, we found that the stress-strain curve of the sciatic nerve specimens was basically in an exponential functional relation at the beginning tensile stress, and then became similar to the linear relationship with the increase of the tensile stress, followed by an approximately exponential functional relation again as the tensile stress was further increased. With the continuously increase of the stress, the stress-strain curves began to have a greater slope with nonlinear change, the sciatic nerve fiber was extended, stretched, until destruction. The elastic limit strain, maximum stress and strain of the rabbit sciatic nerve in the PCBT and PCBET groups were larger than those in the ANT group, indicating that PLGA catheter combined with BMMSCs and ECM transplantation improved the elasticity and strength of animal sciatic nerve in the sciatic nerve injury model. The improved elasticity of the sciatic nerve is conducive to resist deformation and external force and conducive to the repair and regeneration of the injured sciatic nerve. It is believed that the nerve fiber arrangement is disrupted in the sciatic nerve defect rabbit model and the nerve fibers are also damaged, which decreases the tensile mechanical properties. The injured sciatic nerves in the sciatic nerve defect rabbit model were repaired after the treatment with PLGA catheter combined with BMMSCs transplantation or PLGA catheter combined with BMMSCs and ECM transplantation, so the mechanical properties of the sciatic nerves were also restored.

At present, PLGA is a widely used cell culture scaffold in the field of tissue engineering ${ }^{15}$. BMMSCs are a group of stem cells derived from bone marrow, with multipotent property and the self-renewal ability. They can be induced in vitro and in vivo to differentiate into neurons cells with glial phenotype ${ }^{16}$. BMMSCs can secrete large amounts of nutrients that are beneficial for the nerve regeneration, myelination and axonal regeneration ${ }^{17)}$. Studies have shown that the regulation of Schwann cells proliferation by BMMSCs can promote the nerve regeneration ${ }^{18)}$. BMMSCs have convenient source, which can be rapidly proliferated in vitro, and have no transplantation rejection nor the ethical problem, thus they have drawn more and more attentions. At low temperature, extracellular matrix gel is in liquid state, which facilitates the BMMSCs to implant and distribute easily and evenly. Extracellular matrix gel includes fibronectin, laminin, type IV collagen and other extracellular matrix components, which can promote the regeneration of nerve ${ }^{19)}$. ECM gel is the extracellular matrix component secreted by Engelbrath Holm Swarm tumor cell line ${ }^{20)}$, containing collagen, laminin, fibronectin and other ingredients, which is in a liquid state at low temperature, easy to distribute the BMMSCs. When the temperature reaches $37^{\circ} \mathrm{C}, \mathrm{ECM}$ generally becomes the gel, facilitating BMMSCs to move but not leading the BMMSCs to flow outside. Donzelli et $\mathrm{al}^{21)}$ confirmed that ECM can promote nerve regeneration and induce the growth of neurite. In this study, we used PLGA catheter combined with BMMSCs, PLGA catheter combined with BMMSCs and ECM transplantation to treat the sciatic nerve injury animal model. The tensile mechanics, morphology and physiological function of the animal sciatic nerves were restored to some extent, which was consistent with the desired results. PLGA catheter combined with BMMSCs and ECM gel transplantation has good repair effect on the injured sciatic nerve in the animal model of sciatic nerve injury.

The characteristics of this study lied in the tensile mechanical properties analysis after the animal model of sciatic nerve injury was treated with autologous nerve transplantation, with PLGA catheters combined with BMMSCs, or with PLGA catheter combined with BMMSCs and ECM gel transplantation; lied in the establishment of the expression of the stress-strain functional relation of each group by the method of mathematical regression analysis. Using mathematical statistical model to analyze the experimental data of the stress and strain can better clarify the mechanical properties of the sciatic nerve in each group, so as to provide a theoretical basis for the pathogenesis of sciatic nerve injury.

Due to the limited sample size of sciatic nerves and the individual 
difference of rabbit sciatic nerves, the experimental data might have some discreteness, but it still has certain reference value for the clinical repair of the injured sciatic nerves.

\section{Acknowledgement}

This work was supported by the Science and Technology Development Plan of Jilin Province, China (20110492).

\section{Conflict of Interest}

The authors declare no conflict of interest.

\section{References}

1. Cheung HY, Lau KT, Lu TP and Hui D. A critical review on polymer-based bio-engineered materials for scaffold development. Composites Part B: Engineering 38: 291-300, 2007

2. Lu L, Peter SJ, Lyman MD, Lai HL, Leite SM, Tamada JA, Uyama S, Vacanti JP, Langer R and Mikos AG. In vitro and in vivo degradation of porous poly(DL-lactic-co-glycolic acid) foams. Biomaterials 21: 1837-1845, 2001

3. Hoban DB, Howard L and Dowd E. GDNF-secreting mesenchymal stem cells provide localized neuroprotection in an inflammation-driven rat model of Parkinson's disease. Neuroscience 303: 402-411, 2015

4. Jo H, Jung M, Seo DJ and Park DJ. The effect of rat bone marrow derived mesenchymal stem cells transplantation for restoration of olfactory disorder. Biochem Biophys Res Commun 467: 395-399, 2015

5. García-Pérez MM, Martínez-Rodríguez HG, López-Guerra GG, Soto-Domínguez A, Said-Fernández SL, Morales-Avalos R, Elizondo-Omaña RE, Montes-de-Oca-Luna R, Guzmán-López S, Castillo-Galván ML, Mendoza-Lemus OF and Vílchez-Cavazos F. A modified chemical protocol of decellularization of rat sciaticnerve and its recellularization with mesenchimal differentiated schwannlike cells: morphological and functional assessments. Histol Histopathol 32: 779-792, 2017

6. Huang YC, Huang CC, Huang YY and Chen KS. Surface modification and characterization of chitosan or PLGA membrane with laminin by chemical and oxygen plasma treatment for neural regeneration. J Biomed Mater Res A 82: 842-851, 2007

7. Moore MJ, Friedman JA, Lewellyn EB, Mantila SM, Krych AJ, Ameenuddin S, Knight AM, Lu L, Currier BL, Spinner RJ, Marsh RW, Windebank AJ and Yaszemski MJ. Multiple-channel scaffolds to promote spinal cord axonregeneration. Biomaterials 27: 419-429, 2006

8. Liu W, Cui L and Cao Y. Bone reconstruction with bone marrow stromal cells. Methods Enzymol 420: 362-380, 2006
9. Kurwale NS, Suri V, Srivastava A, Suri A, Mohanti S, Yadav P, Sharma MC and Sarkar C. Role of bone marrow derived pluripotent stem cells in peripheral nerve repair in adult rats: A morphometric evaluation. J Neurosci Rural Pract 6: 152-159, 2015

10. Zhou LN, Zhang JW, Liu XL and Zhou LH. Co-graft of bone marrow stromal cells and schwann cells Into acellular nerve scaffold for sciatic nerve regeneration in rats. J Oral Maxillofac Surg 73: 16511660,2015

11. Tlili S, Gay C, Graner F, Marcq P, Molino F and Saramito P. Colloquium: Mechanical formalisms for tissue dynamics. Eur Phys J E Soft Matter 38: 121, 2015

12. Pawlaczyk M, Lelonkiewicz M and Wieczorowski M. Age-dependent biomechanical properties of the skin. Postepy Dermatol Aler-gol 30: 302-306, 2013

13. Zhang ZJ, Li YJ, Liu XG, Huang FX, Liu TJ, Jiang DM, Lv XM and Luo M. Human umbilical cord blood stem cells and brain-derived neurotrophic factor for optic nerve injury: a biomechanical evaluation. Neural Regen Res 10: 1134-1138, 2015

14. Wang Y, Li ZW, Luo M, Li YJ and Zhang KQ. Biological conduits combining bone marrow mesenchymal stem cells and extracellular matrix to treat long-segment sciatic nerve defects. Neural Regen Res 10: 965-971, 2015

15. Kim WS, Vacanti JP, Cima L, Mooney D, Upton J, Puelacher WC and Vacanti CA. Cartilage engineered in predetermined shapes employing cell transplantation on synthetic biodegradable polymers. Plast Reeonstr Surg 94: 233-237, 1994

16. Chen X, Wang XD, Chen G, Lin WW, Yao J and Gu XS. Study of in vivo differentiation of rat bone marrow stromal cells into schwann cell-like cells. Microsurgery 26: 111-115, 2006

17. Lin W, Chen X, Wang X, Liu J and Gu X. Adult rat bone marrow stromal cells differentiate into Schwann cell-like cells in vitro. In Vitro Cell Dev Biol Anim 44: 31-40, 2008

18. Shimizu S, Kitada M, Ishikawa H, Itokazu Y, Wakao S and Dezawa M. Peripheral nerve regeneration by the in vitro differentiated-human bone marrow stromal cells with Schwann cell property. Biochem Biophys Res Commun 359: 915-920, 2007

19. Wang J, Ding F, Gu Y, Liu J and Gu X. Bone marrow mesenchymal stem cells promote cell proliferation and neurotrophic function of Schwann cells in vitro and in vivo. Brain Res 1262: 7-15, 2009

20. Orkin RW, Gehron P, McGoodwin EB, Martin GR, Valentine T and Swarm R. A murine tumor producing a matrix of basement membrane. J Exp Med 145: 204-220, 1977

21. Donzelli R, Maiuri F, Piscopo GA, de Notaris M, Colella A and Divitiis E. Role of extracellular matrix components in facial nerve regeneration: an experimental study. Neurol Res 28: 794-801, 2006 